been exposed to herbicides their wives have had more pregnancies where the outcome was unfavourable, that is spontaneous abortions and child born deformed, than was observed in a control population.

The increase in chromosome aberrations and sister chromatid exchange observed in adults exposed to herbicides and in their children was assessed using standard nonbanding cytogenetic techniques. As the published literature on this subject is extremely controversial ${ }^{6}$, it was felt that these findings required urgent confirmation in other laboratories.

Much of the work presented by Vietnamese scientists at the conference was in many ways only a first look at the problem.

More work is required to validate their findings and cooperation on an international basis to facilitate the programme is being sought. International aid agencies, such as WHO and those in the UN concerned with the environment, have an important part to play and will be called upon to help.

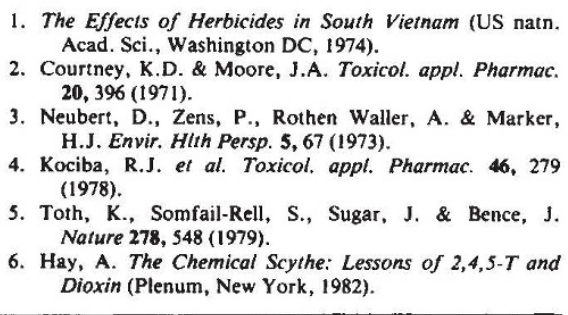

\title{
Supernova
}

\section{Carbon detonation redux}

\section{from J. Craig Wheeler}

A DECADE ago carbon detonation was all the rage among supernova theorists. The idea was that the characteristic burst of light and other radiation that astronomers label a type I supernova came from a star whose core of degenerate carbon suddenly underwent explosive nuclear burning. The situation was thought to arise in highly evolved stars of mass 4-8 $M_{\odot}$ after they had lost their hydrogen envelope in the stellar wind.

The notion that 4-8 $M_{\odot}$ stars were the site of the 'carbon detonation supernova' permeated the general astronomical community at about the time the experts were deciding that carbon probably does not detonate and that 4-8 $M_{\odot}$ stars may not ignite carbon anyway. However, new concepts of the nature of type I supernovae and novel numerical procedures have brought the question of the nature of degenerate carbon ignition to the forefront of supernova research again. In a recent paper, the first hydrodynamical calculations of carbon models are carried out by W. David Arnett, and Ewald Muller makes an important new contribution towards understanding the complex problem of degenerate carbon ignition (Muller \& Arnett Astrophys. J. Lett. 261, L109; 1982). This new work reinforces the idea that degenerate carbon ignition will lead to the subsonic combustion of a major fraction, but not all, of the star and to complete disruption with no neutron star remnant.

In the mid 1970 s the realization slowly came that highly degenerate carbon (that is, a highly compressed form of carbon that departs from the classical equation of state in its behaviour) would not burn by means of a self-propagating supersonic detonation wave. This conclusion did not come easily because a detonation will propagate in appropriate stellar conditions once it is initiated. The problem is that the Fermi energy in carbon at densities in excess of $10^{9} \mathrm{~g} \mathrm{~cm}^{-3}$ is so high that the ignition and in- cineration of a small portion of the centre of the star does not provide enough overpressure to drive a sufficiently strong shock. The conclusion is strengthened by consideration of geometrical factors in the spherical expansion of the small central portion where ignition begins. It appears, rather, that degenerate carbon, though extremely volatile, burns by means of a subsonic front which propagates by turbulent exchange of heat between incinerated and unburned matter.

Few modern problems in stellar evolution do not founder on the shoals of turbulence. Carbon burning may well propagate by turbulent interchange but one is left with the untractable problem of calculating a complex time-dependent hydrodynamical phenomenon. The difficulties are made worse by the complex convectively driven Urca process which may modify significantly the energetics and composition in the brief convective phase that falls between ignition and thermal runaway. The outcome of this process, first suggested by Bohdan Paczynski and calculated in great detail by Icko Iben, is still not clearly understood.

In addition to these difficulties of principle, there was growing circumstantial evidence that 4-8 $M_{\odot}$ stars lose their envelopes to leave stable, cooling, white dwarfs and hence do not even evolve to the condition of carbon ignition. Uncertainty about whether and how to proceed and burgeoning interest in the application of fascinating new physics to the gravitational collapse problem led supernova theorists away from the exploration of degenerate ignition.

The subject returned to the forefront through a conceptual breakthrough in the understanding of type I supernovae. Work by several people on light curves and by Timothy Axelrod on the spectral synthesis of the late-time nebular spectra gave fascinating new evidence that the famous exponential decay in the light curves of type I supernovae is powered by the radioactive decay of ${ }^{56} \mathrm{Ni}$. Nickel is the natural product of degenerate carbon burning.

A model in which the inner portion of a degenerate carbon core is incinerated to nickel, resulting in the total disruption of the star, soon gained popularity. The picture is just that provided by the tentative models of subsonic turbulent carbon buring of the mid 1970s, as pointed out by Ken'ichi Nomoto and others. A crucial underpinning of this class of model is the assumption that supernova distances accord with values of the Hubble constant, $H_{\mathrm{o}} \sim 50 \mathrm{~km} \mathrm{~s}^{-1} \mathrm{Mpc}^{-1}$. If it should be the case, as some evidence indicates, that $H_{\mathrm{o}} \sim 100 \mathrm{~km} \mathrm{~s}^{-1} \mathrm{Mpc}$, the implied lower luminosity requires very little nickel; and carbon burning to produce that small quantity could not account for the observed velocities of type I events. A completely different class of model, perhaps involving gravitational collapse, would then be required.

Nevertheless, the desire to improve our understanding of degenerate carbon ignition has been rekindled. A new ingredient to the numerical approach to this class of problem has been added by Robert Deupree. Deupree has introduced twodimensional hydrodynamical techniques to explore time-dependent non-local convection and has used them to study the degenerate helium flash. Whether even a two-dimensional treatment can adequately represent the complexities of full threedimensional convection has been seriously questioned but Deupree's work has nevertheless shown qualitative departures from previous work. Even if not final, the calculations leave little reason to trust any older results based on one-dimensional hydrodynamics and time-independent mixing length convection.

Muller and Arnett have used twodimensional techniques similar to those of Deupree to re-examine the question of degenerate carbon burning. They find a complex combustion process in which highly non-spherical convective tongues of burned material reach out from the central core accompanied by a cascade of eddies to a size limited by the numerical grid. Though the process is more complex, the net result is approximately the same as simple one-dimensional models assuming turbulent exchange on small length scales. About $1 M \odot$ of nickel is produced and the star is totally disrupted - an outcome quite consistent with the observations of type I supernovae if $H_{\mathrm{o}} \sim 50 \mathrm{~km} \mathrm{~s}^{-1} \mathrm{Mpc}^{-1}$.

The calculations of Muller and Arnett are still limited by grid size and a simplified treatment of strong interactions. They are the first of a new breed, however, and show the way to a new era of study of degenerate carbon burning.

J. Craig Wheeler is in the Department of Astronomy, The University of Texas at Austin, Austin, Texas 78712. 